\title{
Diagnostic performance of serum blood urea nitrogen to creatinine ratio for distinguishing prerenal from intrinsic acute kidney injury in the emergency department
}

\author{
Guillaume Manoeuvrier ${ }^{1}$, Kalyane Bach-Ngohou², Eric Batard ${ }^{3,4}$, Damien Masson² and David Trewick ${ }^{3,4^{*}}$
}

\begin{abstract}
Background: The blood urea nitrogen to creatinine ratio (BCR) has been used since the early 1940s to help clinicians differentiate between prerenal acute kidney injury (PR AKI) and intrinsic AKI (I AKI). This ratio is simple to use and often put forward as a reliable diagnostic tool even though little scientific evidence supports this. The aim of this study was to determine whether BCR is a reliable tool for distinguishing PR AKI from I AKI.

Methods: We conducted a retrospective observational study over a 13 months period, in the Emergency Department (ED) of Nantes University Hospital. Eligible for inclusion were all adult patients consecutively admitted to the ED with a creatinine $>133 \mu \mathrm{mol} / \mathrm{L}(1.5 \mathrm{mg} / \mathrm{dL})$.

Results: Sixty thousand one hundred sixty patients were consecutively admitted to the ED. 2756 patients had plasma creatinine levels in excess of $133 \mu \mathrm{mol} / \mathrm{L}, 1653$ were excluded, leaving 1103 patients for definitive inclusion.

Mean age was $75.7 \pm 14.8$ years old, 498 (45\%) patients had PR AKI and 605 (55\%) I AKI. BCR was $90.55 \pm 39.32$ and 91. $29 \pm 39.79$ in PR AKI and I AKI groups respectively. There was no statistical difference between mean BCR of the PR AKI and I AKI groups, $p=0.758$. The area under the ROC curve was 0.5 indicating that BCR had no capacity to discriminate between PR AKI and I AKI.
\end{abstract}

Conclusions: Our study is the largest to investigate the diagnostic performance of BCR. BCR is not a reliable parameter for distinguishing prerenal AKI from intrinsic AKI.

Keywords: Acute kidney injury (AKI), Blood urea creatinine ratio (BCR), Diagnostic performance, Emergency department, Prerenal acute kidney injury

\section{Background}

The blood urea nitrogen to creatinine ratio (BCR) has been used since the early 1940s to help clinicians differentiate between prerenal acute kidney injury (PR AKI) and intrinsic AKI (I AKI) [1]. This ratio is simple to use and is often put forward as a reliable diagnostic tool. Indeed many textbooks of internal medicine, nephrology and critical care continue to advocate the use of BCR even though its usefulness in the diagnosis and clinical management of AKI remains unclear [2-4].

\footnotetext{
* Correspondence: david.trewick@chu-nantes.fr

${ }^{3}$ Department of Emergency Medicine, CHU Nantes, Nantes, France

${ }^{4}$ Service des Urgences, CHU Hotel Dieu, 44000 Nantes, France

Full list of author information is available at the end of the article
}

Under normal conditions, BCR is less than 100 (with urea and creatinine concentrations expressed in $\mathrm{mmol} /$ L) [2-5]. In states of renal hypoperfusion with intact tubular function, blood urea nitrogen (BUN) is considered to rise out of proportion to plasma creatinine concentration, due to avid urea reabsorption by the proximal tubule, the BCR typically becoming $>100$ [6].

One of the first to put forward this tool was Fishberg in 1939 when he observed that "an increase in urea content of the blood may be considerable before the creatinine value rises in prerenal azotemia" [1]. However, as soon as 1947, other investigators found no such relationship [7]. Since then, very few studies (human or animal) have addressed the question and their results are 
conflicting [8-11]. These studies consisted of small series of patients, essentially from intensive care units, the largest of which included only 103 patients and this study was not specifically aimed at investigating BCR [11].

AKI is common in Emergency Departments (ED) [12]. It can be challenging to differentiate prerenal from intrinsic acute kidney injury in this setting. Indeed, the ED physician has a short time frame to make decisions, he may not have access to current medication or baseline creatinine, have incomplete medical history and doesn't have by definition the responsiveness to a fluid challenge.

The BCR is one of the diagnostic tools recommended to ED physicians for differentiating between PR and I AKI, yet it has never been specifically studied in this setting [3].

The aim of this study was to determine whether BCR is a reliable parameter for distinguishing prerenal from intrinsic AKI in a population of patients admitted to hospital via the ED.

\section{Methods}

\section{Study design and patient selection}

We conducted a retrospective observational study over a 13 month period, from 1st of November 2013 to the 30th of November 2014, in the Medical Emergency Department of Nantes University Hospital. Trauma patients were not admitted to this unit. The need for informed consent was waived by the institutional review board of Nantes University Hospital because of the anonymous and purely observational nature of the study.

Eligible for inclusion were all adult patients ( $\geq 16$ years old) consecutively admitted to the ED with a creatinine $>133 \mu \mathrm{mol} / \mathrm{L}(1.5 \mathrm{mg} / \mathrm{dL})$. Patients were excluded if on chronic dialysis or had a prior kidney transplant, stable chronic kidney disease, obstructive AKI, length of hospital stay less than $48 \mathrm{~h}$, no follow up creatinine during the following 7 days of hospitalisation. If a patient was admitted to the ED on several occasions during a 7 day period, only the first creatinine $>133 \mu \mathrm{mol} / \mathrm{L}$ was taken into account, the other admissions were excluded. Patients with no baseline creatinine (lowest creatinine measured 12 months before or after hospital admission) were also excluded.

\section{Laboratory techniques}

Blood samples were collected and centrifuged at $2000 \mathrm{~g}$ for $10 \mathrm{~min}$ at $4{ }^{\circ} \mathrm{C}$ within $1 \mathrm{~h}$ after venipuncture. All biochemical measurements of urea and creatinine were performed in the same laboratory (Laboratory of Clinical Biochemistry, University Hospital of Nantes) respectively with an enzymatic kinetic UV assay and a kinetic colorimetric assay based on the Jaffé method on Cobas c701
(Roche Diagnostics, Mannheim) according to the manufacturer's instructions.

\section{Study definitions}

We defined AKI according to "Kidney Disease: Improving Global Outcomes" (KDIGO) classification scheme based on changes in plasma creatinine during the 7 days after admission (Table 1) [13]. Prerenal AKI (PR AKI) was defined as non-obstructive AKI with a return of plasma creatinine to $110 \%$ (or less) of baseline value during the 7 days following admission. The baseline value of plasma creatinine was the lowest creatinine measured 12 months before or after hospital admission. Intrinsic AKI (I AKI) was defined as non-obstructive AKI that didn't meet the criteria of pre-renal AKI.

\section{Chart review}

Patient's records were reviewed using the hospital admissions and discharges database. Relevant data was entered in a format that could be converted to an Excel spreadsheet for analysis. The following information was obtained for each admission: basic demographics, dates of hospital admission and discharge, admission plasma creatinine, blood urea nitrogen (BUN) levels and BCR, information concerning urinary obstruction, lowest creatinine measured 12 months before/after admission, lowest creatinine measured during the 7 days following admission and presence or absence of AKI using KDIGO classification.

\section{Statistical analysis}

All statistical analysis was performed using GraphPad Prism Software (La Jolla, CA, USA). Quantitative data were expressed as mean \pm standard deviation (SD). Comparison of biochemical and clinical data between prerenal AKI (PR AKI) and intrinsic AKI (I AKI) patients was done using unpaired t-tests. Predictive

Table 1 Staging of Acute Kidney Injury (AKI) according to KDIGO criteria

\begin{tabular}{ll}
\hline Stage & Criteria \\
\hline Stage 1 & $\begin{array}{l}\text { One of the following } \\
\cdot\end{array}$ \\
& $\cdot$ Serum creatinine increased $1.5-1.9$ times baseline \\
& $\cdot$ Urinary output $<0.5 \mathrm{~mL} / \mathrm{kg} / \mathrm{h}$ for $6-12 \mathrm{~h}$ \\
Stage 2 & $\cdot$ Serum creatinine increase $2.0-2.9$ times baseline \\
& $\cdot$ Urinary output $<0.5 \mathrm{~mL} / \mathrm{kg} / \mathrm{h}$ for more than $12 \mathrm{~h}$ \\
Stage 3 & $\cdot$ Serum creatinine increase $>3$ times baseline \\
& $\cdot$ Serum creatinine increases to $>353.6 \mu$ mol $/ \mathrm{L}(4.0 \mathrm{mg} / \mathrm{dL})$ \\
& $\cdot$ Initiation of renal replacement therapy \\
& $\cdot$ Urinary output $<0.3 \mathrm{~mL} / \mathrm{kg} / \mathrm{h}$ during more than $24 \mathrm{~h}$ \\
& $\cdot$ Anuria for more than $12 \mathrm{~h}$ \\
\hline
\end{tabular}


performance of $\mathrm{BCR}$ in terms of specificity and sensitivity was performed using Receiver Operating Characteristic (ROC) analysis. For all analyses, a $P$ value $<0.05$ was considered to be statistically significant.

\section{Results}

During the study period, 60,160 patients $\geq 16$ years old were consecutively admitted to the Emergency Department of Nantes University Hospital. Creatinine levels were measured 28,149 times for 26,299 patients. Two thousand seven hundred and fifty six had plasma creatinine levels in excess of $133 \mu \mathrm{mol} / \mathrm{L}$ but 1653 were excluded because they met one or more exclusion criteria, leaving 1103 patients for definitive inclusion (Fig. 1).

The demographic characteristics of the 1103 included patients are presented in Table 2. Mean age was $75.7 \pm 14.8$ years old (range [16-103]; median 80), 693 $(62.8 \%)$ were male. According to our definitions, 498 (45\%) patients had prerenal AKI (PR AKI) and 605 (55\%) intrinsic AKI (I AKI). The PR AKI and I AKI groups do not differ in terms of age $(p=0.518)$ (Table 2). Patients are classified according to their stage of AKI in Table 3.

At admission, mean blood urea nitrogen (BUN) concentration was $18.1 \pm 9.5 \mathrm{mmol} / \mathrm{L}$ (range [4.8-68.6]; median 15.6) and $19.8 \pm 9.6 \mathrm{mmol} / \mathrm{L}$ (range [4.0-68.7]; median 15.9) in PR AKI and I AKI groups respectively $(p=0.003)$ (Fig. 2a and Table 2). At admission, mean plasma creatinine concentration was $209.6 \pm 118.3 \mu \mathrm{mol} / \mathrm{L}$ (range [134-1376]; median 177) and $232.1 \pm 124.8 \mu \mathrm{mol} / \mathrm{L}$ (range [134-1089]; median 190) in the PR AKI and I AKI groups respectively $(p=0.002)$ (Fig. $2 \mathrm{~b}$ and Table 2$)$.

During the 7 days following admission, the patient's lowest plasma creatinine concentrations were selected and compared with their baseline creatinines (Fig. 2c and Table 2). In the PR AKI group, the mean lowest creatinine concentrations during the 7 days follow-up was $118.0 \pm 65.2 \mu \mathrm{mol} / \mathrm{L}$ (range [30-606]; median 105). The mean difference with plasma creatinine concentrations at admission was $91.6 \mu \mathrm{mol} / \mathrm{L}$. In the intrinsic AKI group, the mean lowest creatinine concentrations during the 7 days follow-up was $162.4 \pm 96.6 \mu \mathrm{mol} / \mathrm{L}$ (range [32-832]; median 138). The mean difference with plasma creatinine concentrations at admission was $69.7 \mu \mathrm{mol} / \mathrm{L}$. Mean difference between baseline and lowest 7 day follow-up creatinine was significantly lower in the PR AKI group than in I AKI group $(p<0.001)$ (Fig. 2c).

The distribution of the blood urea nitrogen to creatinine ratio (BCR) in the 2 groups is shown in Fig. 3. At admission, BCR was $90.6 \pm 39.3$ (range [24.4-262.7]; median 83.0) and $91.3 \pm 39.8$ (range [9.8-269.1]; median 81.8) in PR AKI and I AKI groups respectively. There was no statistical difference between mean BCR of the prerenal AKI group and the intrinsic AKI group, $p=0.758$ (Fig. 3 and Table 2).

The prediction of the capacity of BCR to correctly classify AKI as intrinsic or prerenal was further tested by a Receiver Operating Characteristic (ROC) curve. The area under the curve was 0.5 indicating that the $\mathrm{BCR}$

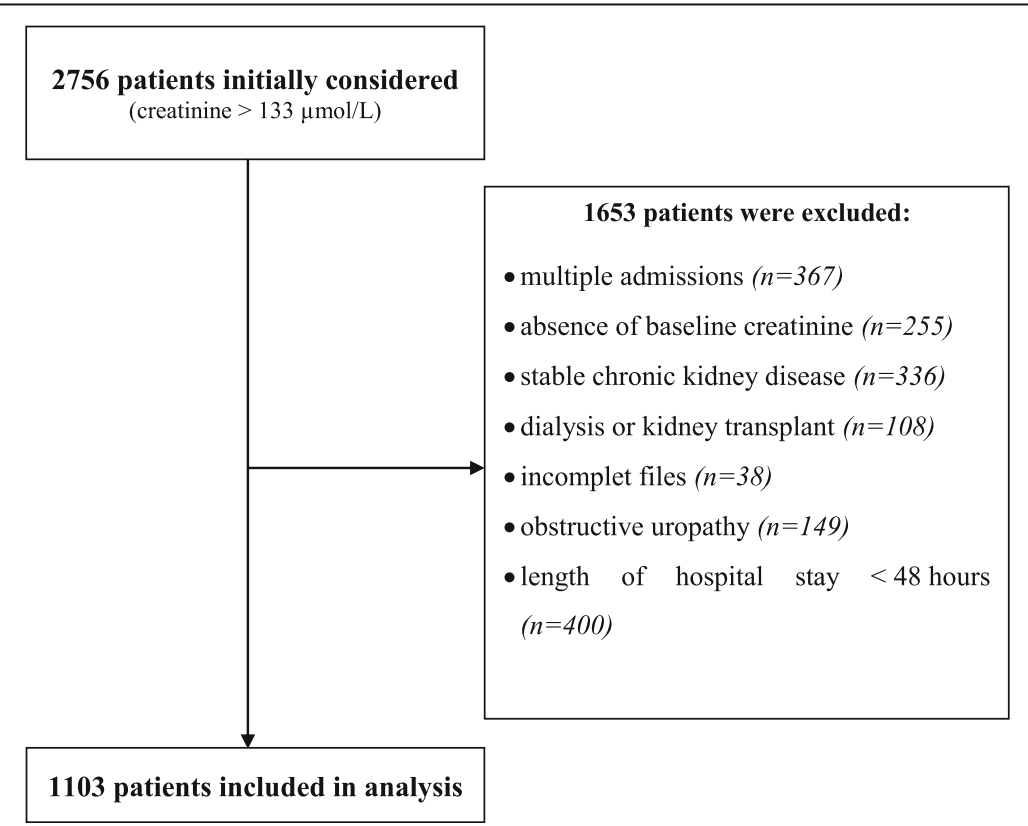

Fig. 1 Study Flow-chart - Patients admitted with plasma creatinine $>133 \mu \mathrm{mol} / \mathrm{L}$ to the Medical Emergency Department of Nantes University Hospital from 1st of November 2013 to the 30th of November 2014 
Table 2 Demographic and biological characteristics of patients with intrinsic AKI (I-AKI) and prerenal AKI (PR-AKI). Results are presented as number of patients and proportions when appropriate or means ( \pm standard deviation)

\begin{tabular}{|c|c|c|c|}
\hline & $\begin{array}{l}\text { I-AKI } \\
(N=605)\end{array}$ & $\begin{array}{l}\text { PR-AKI } \\
(N=498)\end{array}$ & $P$ \\
\hline Age (years) & $75.7(14.3)$ & $75.7(15.3)$ & 0.518 \\
\hline Gender, $\mathrm{n}$ male (\%) & $370(61.2)$ & $323(64.9)$ & \\
\hline Plasma creatinine at admission $(\mu \mathrm{mol} / \mathrm{L})$ & $232.1(124.8)$ & $209.6(118.3)$ & 0.002 \\
\hline Lowest plasma creatinine during the 7 days following admission ( $\mu \mathrm{mol} / \mathrm{L}$ ) & $162.4(96.6)$ & $118.0(65.2)$ & $<0.001$ \\
\hline Blood urea nitrogen at admission $(\mathrm{mmol} / \mathrm{L})$ & $19.8(9.6)$ & $18.1(9.5)$ & 0.003 \\
\hline Blood urea nitrogen to creatinine ratio at admission $(\mathrm{mmol} / \mathrm{L} / \mathrm{mmol} / \mathrm{L})$ & $91.3(39.8)$ & $90.6(39.3)$ & 0.758 \\
\hline
\end{tabular}

had no capacity to discriminate between prerenal and intrinsic AKI (Fig. 4). At the threshold of 100 commonly used to distinguish PR AKI (BCR > 100) from I AKI $(B C R<100)$, the sensitivity was $70.1 \%$ and the specificity $32.6 \%$.

The distribution of patients with a BCR $<100$ or $>100$ was further analysed and compared to renal recovery (Table 4).

If prerenal AKI was redefined as a return of serum creatinine to $110 \%$ of baseline in $72 \mathrm{~h}$ (rather than 7 days), 418 (38\%) patients could be analysed. These are the patients that had BUN and creatinine measured precisely at $72 \mathrm{~h}$. Then 166 (39.7\%) patients had PR AKI and 252 (60.3\%) I AKI. At admission, mean BCR was $88.4 \pm 39.6$ (range [24.4-229.8]; median 82.8) and $95.3 \pm 42.7$ (range [9.8-269.1]; median 86.2) in PR AKI and I AKI groups respectively. There was no statistical difference between mean BCR of the prerenal AKI group and the intrinsic AKI group, $p=0.094$. The area under the ROC curve was 0.55 .

Analysis of patients presenting with acute heart failure ( $n=191)$, typically associated with increased BCR, showed that 33\% had PR AKI and 67\% had I AKI, mean BCR was $98.3 \pm 38$ (range [37.3-250.6]; median 90.2).

\section{Discussion}

Our study is the largest concerning the diagnostic performance of $\mathrm{BCR}$ for differentiating prerenal from intrinsic AKI. It is the first to specifically investigate BCR in an unselected population of patients admitted to the Emergency Department. We have found that BCR had no overall discriminative capacity in this setting, no matter what threshold of BCR is chosen.

Even though the rationale underlying the use of BCR is seducing, our results show that it simply doesn't work

Table 3 Staging of AKI patients according to KDIGO criteria

\begin{tabular}{llll}
\hline & Stage 1 & Stage 2 & Stage 3 \\
\hline Number of AKI patients (\%) & $334(30.3)$ & $576(52.2)$ & $193(17.5)$ \\
PR AKI (\%) & 58.7 & 42.5 & 29.5 \\
I AKI (\%) & 41.3 & 57.5 & 70.5 \\
\hline
\end{tabular}

in the real world. Indeed, many factors are known to modify BCR independently of effective circulating volume. Gastro intestinal bleeding, a high protein diet, the catabolic effects of fever, trauma, infection, thyrotoxicosis, drugs such as tetracycline or corticosteroids, all increase protein turnover resulting in increased hepatic production of urea and increase $\mathrm{BCR}[5,6]$. Conversely, in osmotic diuresis and with the use of acetazolamide, proximal tubular reabsorption of salt and water is impaired leading to an increase in excreted urea and a decrease in BCR even in states of hypovolemia. BCR also decreases in patients with liver failure or protein malnutrition due to lower levels of BUN [5, 6]. One can also speculate that AKI is probably due to functional and intrinsic disease coexisting in different proportions, in a given patient at a given time. Indeed, it is assumed that a continuum exists which leads from prerenal to intrinsic AKI, the proportion of each changing over time [14]. It can then easily be assumed that BCR would only be reliable if the underlying disease was $100 \%$ prerenal or $100 \%$ intrinsic, which is probably rarely the case.

We chose a "cut off creatinine" for inclusion of patients at $133 \mu \mathrm{mol} / \mathrm{L}$. The reason for this was twofold: $1 /$ This threshold is recognized as the highest creatinine level one can have with a normal glomerular filtration rate (GFR) ie $75 \mathrm{~mL} / \mathrm{min}$ per $1.73 \mathrm{~m}^{2}$ [15]. Indeed, a young (20-29 years old) black male with a creatinine of $133 \mu \mathrm{mol} / \mathrm{L}$ would have a normal GFR [15]. However, all creatinine levels above $133 \mu \mathrm{mol} / \mathrm{L}$ are associated with altered GFRs. By including only patients with creatinine levels greater than $133 \mu \mathrm{mol} / \mathrm{L}$ we were sure to only include patients with renal failure. 2/ If we had included all 26,229 patients with a measured creatinine, we would have selected many healthy individuals admitted to hospital for the first time. Many patients would have met our exclusion criteria because of a short hospital stay $(<48 \mathrm{~h})$ and or no baseline creatinine.

A limitation that affects most studies on AKI, is the absence of a baseline creatinine for all patients. Indeed, the KDIGO classification scheme requires a baseline creatinine level to define AKI. In the absence of such values a method of "imputation" or "back calculation" by reversing the "Modification of Diet in Renal Disease" (MDRD) equation 

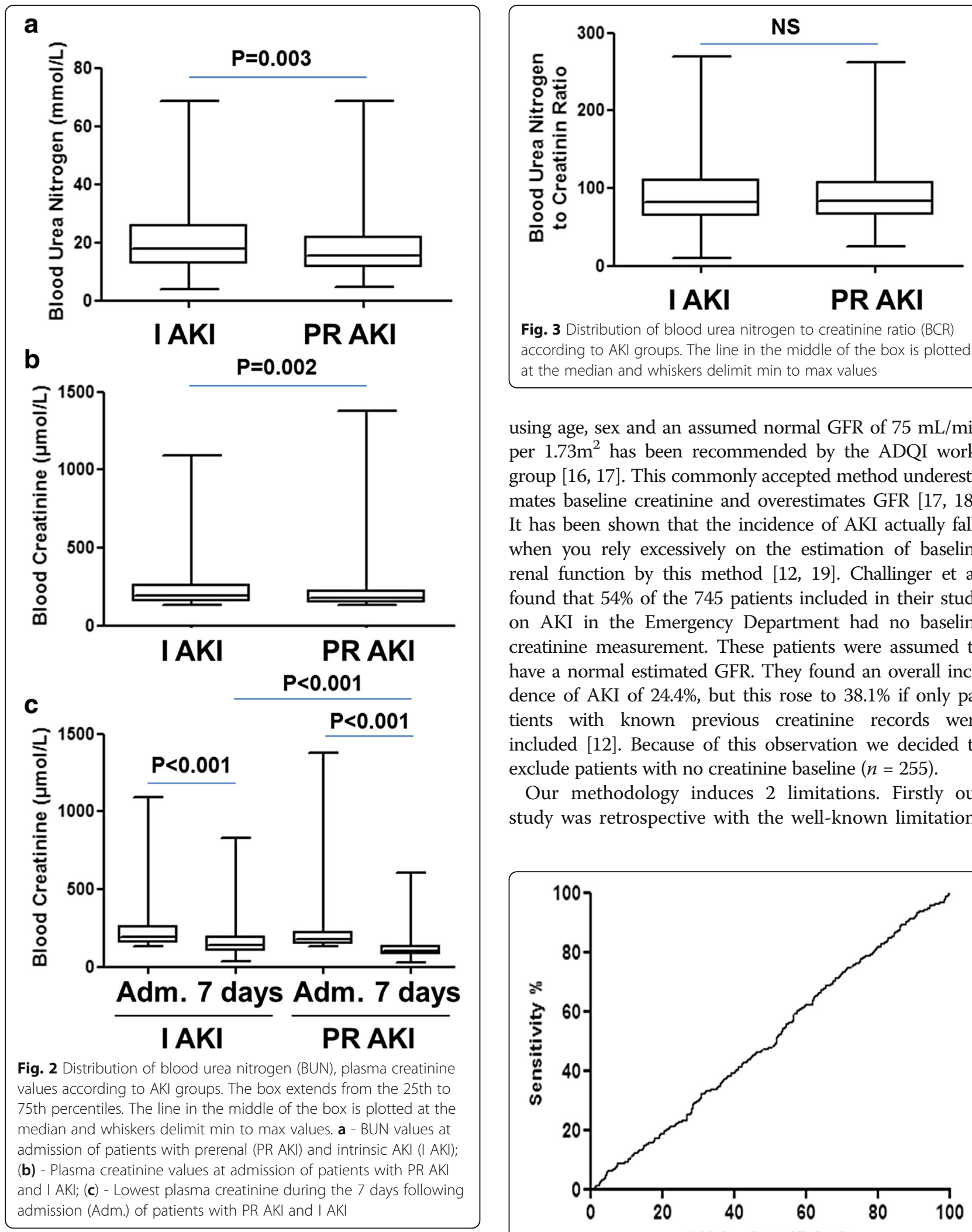

Fig. 3 Distribution of blood urea nitrogen to creatinine ratio (BCR) according to AKI groups. The line in the middle of the box is plotted at the median and whiskers delimit min to max values

using age, sex and an assumed normal GFR of $75 \mathrm{~mL} / \mathrm{min}$ per $1.73 \mathrm{~m}^{2}$ has been recommended by the ADQI workgroup $[16,17]$. This commonly accepted method underestimates baseline creatinine and overestimates GFR [17, 18]. It has been shown that the incidence of AKI actually falls when you rely excessively on the estimation of baseline renal function by this method $[12,19]$. Challinger et al. found that $54 \%$ of the 745 patients included in their study on AKI in the Emergency Department had no baseline creatinine measurement. These patients were assumed to have a normal estimated GFR. They found an overall incidence of AKI of $24.4 \%$, but this rose to $38.1 \%$ if only patients with known previous creatinine records were included [12]. Because of this observation we decided to exclude patients with no creatinine baseline $(n=255)$.

Our methodology induces 2 limitations. Firstly our study was retrospective with the well-known limitations

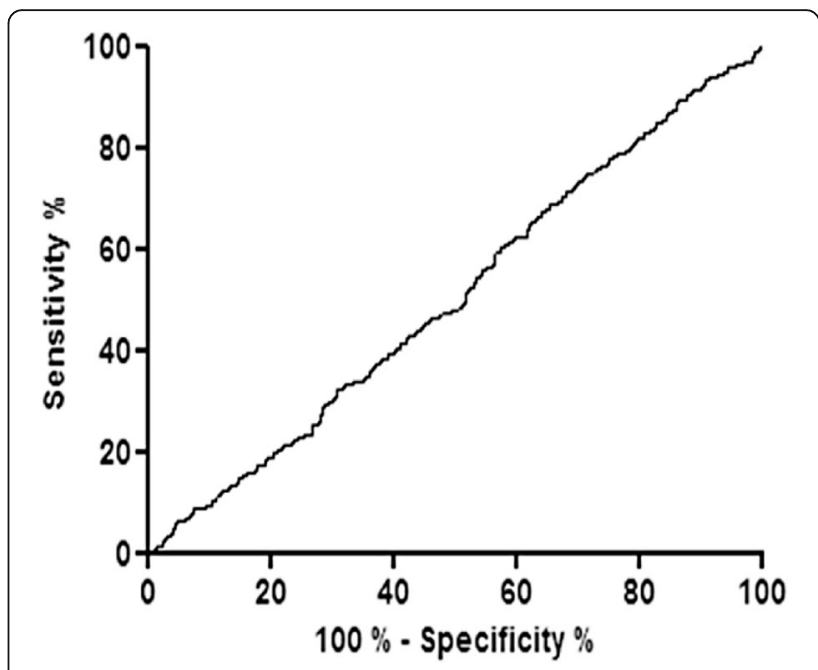

Fig. 4 Receiver operating curve analysis of predictive performance of blood urea nitrogen to creatinine ratio $(B C R)$ 
Table 4 Distribution of prerenal and intrinsic AKI patients according to whether $\mathrm{BCR}<$ or $>100$

\begin{tabular}{lll}
\hline & BCR $<100$ & BCR $>100$ \\
\hline Number of patients (\%) & $754(68.4)$ & $349(31.6)$ \\
PR AKI (\%) & 45.9 & 43.6 \\
I AKI (\%) & 54.1 & 56.4 \\
\hline
\end{tabular}

of such studies and in particular it was not possible for us to assess the contribution of the "urine output", component of the KDIGO criteria. Secondly there is no widely accepted definition of prerenal AKI [6]. The common observation is that it is a reversible condition, but diagnostic criteria such as the amount, nature and duration of fluid resuscitation, time frame for reversibility, or target creatinine improvement have not been addressed. We defined prerenal AKI as a return of serum creatinine to $110 \%$ (or less) of the baseline value during the 7 days following admission. In their review of AKI, Bagshaw et al. reported that time frames for defining reversibility ie PR AKI, varied considerably from $48 \mathrm{~h}$ to 7 days or more and sometimes were not reported at all [8]. The shorter $(48-72 \mathrm{~h})$ is the most published time frame but concerns almost exclusively patients from intensive care units, where blood samples are drawn every day which is not necessarily the case for most patients, such as ours, admitted to medical wards [6]. By choosing a $72 \mathrm{~h}$ time frame for reversibility we would have had to exclude many patients who didn't have blood samples precisely on day 3 . We did however carry out a subgroup analysis of these patients. We found 418 (38\%) patients that had BUN and creatinine measured at 72 h. Again there was no statistical difference between mean BCR of the prerenal and the intrinsic AKI groups, the area under the ROC curve was 0.55 .

Separating prerenal from intrinsic AKI remains challenging. No biological marker, whether old school (fractional excretion of sodium or urea, urinary sodium (UNa,) urine-to-plasma creatinine ratio (U:PCr), etc) or new biomarkers such as Cystatin C, urinary neutrophil gelatinase-associated lipocalin (N-GAL), has clearly demonstrated a capacity to correctly classify AKI $[6,14,20,21]$. On the ED, the importance of taking a complete medical history, obtaining the list of current potentially nephrotoxic medication, assessing hemodynamic status and if required initiating a fluid challenge, still remains "cornerstone" management of AKI.

\section{Conclusions}

Our study is the largest to investigate the diagnostic performance of BCR. We found that BCR was not a reliable parameter for distinguishing prerenal AKI from intrinsic AKI in a population of patients admitted to hospital via the Emergency Department.

\section{Abbreviations}

AKI: Acute kidney injury; BCR: Blood urea nitrogen to creatinine ratio; BUN: Blood urea nitrogen; ED: Emergency department; GFR: Glomerular filtration rate; I AKI: Intrinsic acute kidney injury; KDIGO: Kidney disease improving global outcomes; MDRD: Modification of diet in renal disease; N-GAL: Neutrophil gelatinase associated lipocalin; PR AKI: Prerenal acute kidney injury; ROC: Receiver operating characteristic

\section{Acknowledgements}

Not applicable.

\section{Funding}

No funding was obtained for this study.

\section{Availability of data and materials}

The datasets used and/or analysed during the current study are available from the corresponding author on reasonable request.

\section{Authors' contributions}

GM: Conception / design/ drafting. KB: Statistics, revising article. EB: Conception, statistics, revising article. DM: Statistics, revising article. DT: Conception/design/drafting/revising article. All authors have read and approved the manuscript.

\section{Competing interests}

The authors declare that they have no competing interests.

\section{Consent for publication \\ Not applicable.}

\section{Ethics approval and consent to participate}

The need for informed consent was waived by the institutional review board of Nantes University Hospital because of the anonymous and purely

observational nature of the study.

\section{Publisher's Note}

Springer Nature remains neutral with regard to jurisdictional claims in published maps and institutional affiliations.

\section{Author details}

${ }^{1}$ Department of Medicine, Clinique Jules Verne, Nantes, France. ${ }^{2}$ Department of Biology, Laboratory of Clinical Biochemistry, CHU Nantes, Nantes, France. ${ }^{3}$ Department of Emergency Medicine, CHU Nantes, Nantes, France. ${ }^{4}$ Service des Urgences, CHU Hotel Dieu, 44000 Nantes, France.

Received: 1 February 2017 Accepted: 17 May 2017

Published online: 25 May 2017

References

1. Fishberg AM. Hypertension and nephritis, in Lea \& Febiger (eds): Philadelphia, 1939.

2. Lin J, Denker BM. Azotemia and urinary abnormalities. In: Kasper DL, editor. Harrison's principles of internal medicine. 19th ed. New York: McGraw Hill Education; 2015. p. 289-95.

3. Wolfson AB. Renal failure. In: Marx JA, Rosen P, editors. Rosen's emergency medicine: concepts and clinical practice. 8th ed. Philadelphia: Elsevier/Saunders; 2014. p. 1291-311.

4. Steddon S, Chesser A, Cunningham J, Ashman N. Acute kidney injury; in Singh P, Fenech LE (eds): Oxford Handbook of Nephrology and Hypertension, 2nd ed. New York: Oxford University Press, 2014; pp87-189.

5. Baum N, Dichoso CC, Carlton CE. Blood urea nitrogen and serum creatinine. Urology. 1975;5:583-8.

6. Macedo E, Mehta RL. Prerenal failure: from old concepts to new paradigms. Curr Opin Crit Care. 2009;15:467-73.

7. Bell ET, Knutson RC. Extrarenal azotemia and tubular disease. JAMA. 1947;135:441-6.

8. Bagshaw SM, Langenberg C, Bellomo R. Urinary biochemistry and microscopy in septic acute renal failure: a systematic review. Am J Kid Dis. 2006;48:695-705.

9. Lumeij JT. Plasma urea, creatinine and uric acid concentrations in response to dehydration in racing pigeons. Avian Pathol. 1987;16:377-82. 
10. Fushimi K, Shichiri M, Marumo F. Decreased Fractional Excretion of Urate as an Indicator of Prerenal Azotemia. Am J Nephrol. 1990;10:489-94.

11. Carvounis CP, Nisar S, Guro-Razuman S. Significance of the fractional excretion of urea in the differential diagnosis of acute renal failure. Kidney Int. 2002;62:2223-9.

12. Challiner R, Ritchie JP, Fullwood C, Loughnan P, Hutchison AJ. Incidence and consequence of acute kidney injury in unselected emergency admissions to a large acute UK hospital trust. BMC Nephrol. 2014;15:84.

13. Kidney Disease: Improving Global Outcomes (KDIGO). Acute kidney injury: KDIGO clinical practice guideline for acute kidney injury. Kid Int. 2012;2:S1-S138.

14. Darmon M, Vincent F, Dellamonica J, et al. Diagnostic performance of fractional excretion of urea in the evaluation of critically ill patients with acute kidney injury: a multicenter cohort study. Crit Care. 2011;15:R178.

15. Bellomo R, Ronco C, Kellum JA, et al. Acute renal failure - definition, outcome measures, animal models, fluid therapy and information technology needs: the Second International Consensus Conference of the Acute Dialysis Quality Initiative (ADQI) Group. Crit Care. 2004;8:204-12.

16. Nation Kidney Foundation. KDOQI clinical practice guidelines for chronic kidney disease: Evaluation, classification and stratification. Am J Kidney Dis. 2002;39:S1-S266.

17. Thomas ME, Blaine C, Dawnay A, et al. The definition of acute kidney injury and its use in practice. Kidney Int. 2015;87:62-73.

18. Pickering JW, Endre ZH. Back-calculating baseline creatinine with MDRD misclassifies acute kidney injury in the intensive care unit. Clin J Am Soc Nephrol. 2010;5:1165-73.

19. Zeng X, McMahon GM, Brunelli SM, Bates DW, Waikar SS. Incidence, outcomes, and comparaisons across definitions of AKI in hospitalized individuals. Clin J Am Soc Nephrol. 2014;9:12-20.

20. Rachoin J-S, Daher R, Moussallem C, et al. The fallacy of the BUN:creatinine ratio in critically ill patients. Nephrol Dial Transplant. 2012;27:2248-54.

21. Vammassenhove J, Vanholder R, Nagler E, Van Biesen W. Urinary and serum biomarkers for the diagnosis of acute kidney injury: an in-depth review of the literature. Nephro Dial Transplant. 2013;28:254-73.

\section{Submit your next manuscript to BioMed Central and we will help you at every step:}

- We accept pre-submission inquiries

- Our selector tool helps you to find the most relevant journal

- We provide round the clock customer support

- Convenient online submission

- Thorough peer review

- Inclusion in PubMed and all major indexing services

- Maximum visibility for your research

Submit your manuscript at www.biomedcentral.com/submit

CBiomed Central 